\title{
An Atypical Long-Term Thiamine Treatment Regimen for Wernicke Encephalopathy
}

\author{
Samra Shoaib, MD; Mehnaz Hyder, MD; and Margaret May, MD
}

\begin{abstract}
A patient with rapidly changing mental status responded to treatment with intramuscular or IV thiamine but not oral dosages, suggesting that presentation of thiamine deficiency can be highly variable, which can complicate the correct diagnosis and treatment.
\end{abstract}

W ernicke-Korsakoff syndrome is a cluster of symptoms attributed to a disorder of vitamin Bl (thiamine) deficiency, manifesting as a combined presentation of alcohol-induced Wernicke encephalopathy (WE) and Korsakoff syndrome (KS). ${ }^{1}$ While there is consensus on the characteristic presentation and symptoms of WE, there is a lack of agreement on the exact definition of KS. The classic triad describing WE consists of ataxia, ophthalmoplegia, and confusion; however, reports now suggest that a majority of patients exhibit only 1 or 2 of the elements of the triad. KS is often seen as a condition of chronic thiamine deficiency manifesting as memory impairment alongside a cognitive and behavioral decline, with no clear consensus on the sequence of appearance of symptoms. The typical relationship is thought to be a progression of WE to KS if untreated.

From a mental health perspective, WE presents with delirium and confusion whereas KS manifests with irreversible dementia and a cognitive deterioration. Though it is commonly taught that KS-induced memory loss is permanent due to neuronal damage (classically identified as damage to the mammillary bodies - though other structures have been implicated as well), more recent research suggest otherwise. ${ }^{2}$ A review published in 2018, for example, gathered several case reports and case series that suggest significant improvement in memory and cognition attributed to behavioral and pharmacologic interventions, indicating this as an area deserving of further study. ${ }^{3}$ About 20\% of patients diagnosed with WE by autopsy exhibited none of the classical triad symptoms prior to death. ${ }^{4}$ Hence, these conditions are surmised to be significantly underdiagnosed and misdiagnosed.

Though consensus regarding the appropriate treatment regimen is lacking for $\mathrm{WE}$, a common protocol consists of high-dose parenteral thiamine for 4 to 7 days. ${ }^{5}$ This is usually followed by daily oral thiamine repletion until the patient either achieves complete abstinence from alcohol (ideal) or decreases consumption. The goal is to allow thiamine stores to replete and maintain at minimum required body levels moving forward. In this case report, we highlight the utilization of a long-term, unconventional intramuscular (IM) thiamine repletion regimen to ensure maintenance of a patient's mental status, highlighting discrepancies in our understanding of the mechanisms at play in WE and its treatment.

\section{CASE PRESENTATION}

A 65-year-old male patient with a more than 3-decade history of daily hard liquor intake, multiple psychiatric hospitalizations for WE, and a prior suicide attempt, presented to the emergency department (ED) with increased frequency of falls, poor oral intake, confabulation, and diminished verbal communication. A chart review revealed memory impairment alongside the diagnoses of schizoaffective disorder and WE, and confusion that was responsive to thiamine administration as well as a history of hypertension, hyperlipidemia, osteoarthritis, and urinary retention secondary to benign prostatic hyperplasia (BPH).
Samra Shoaib is a Clinical Extern; Mehnaz Hyder is a Staff Psychiatrist, Inpatient Psychiatry; and Margaret May is a Staff Psychiatrist, Inpatient Psychiatry and Mental Health Evaluation Clinic; all at the Veterans Affairs Palo Alto Health Care System in California. Margaret May also is a Clinical Instructor (Affiliate), Department of Psychiatry and Behavioral Sciences, Stanford University School of Medicine in California. Correspondence: Margaret May (margaret.may@stanford.edu)

Fed Pract. 2020;37(8):405-409. doi: $10.12788 /$ fp.0029 
FIGURE Patient Treatment Timeline

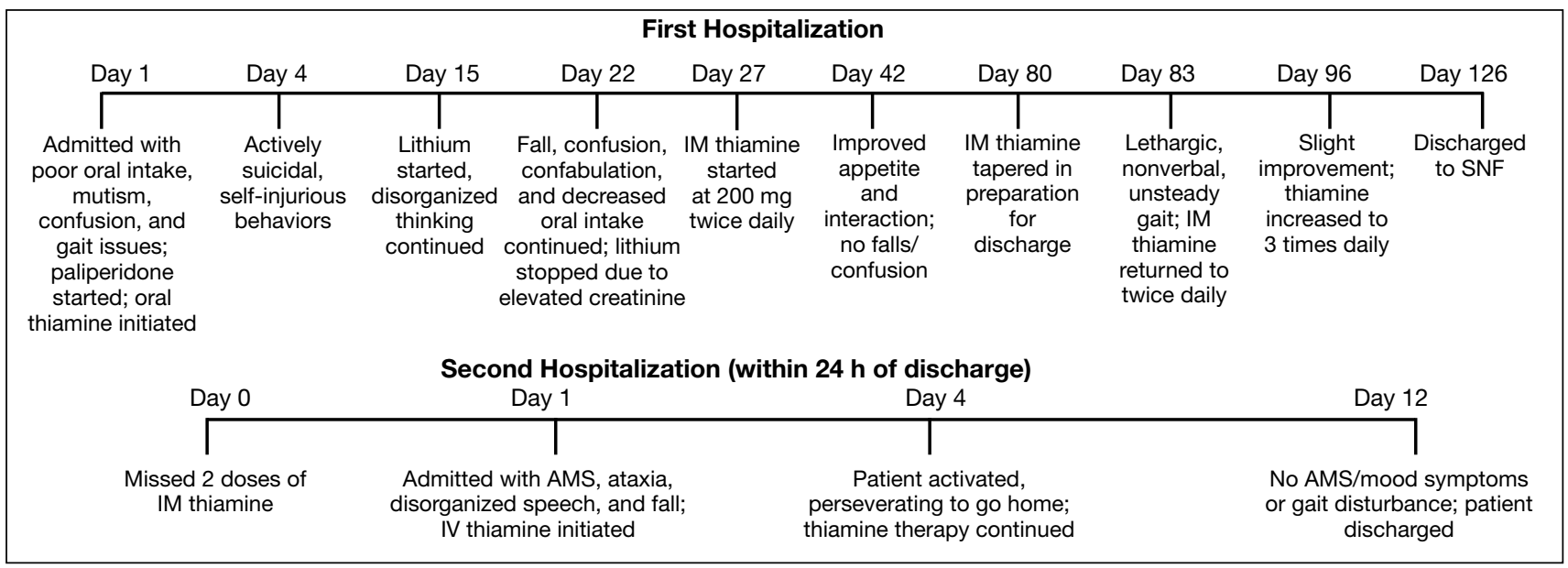

Abbreviations: AMS, altered mental status; IM, intramuscular; SNF, skilled nursing facility.

On examination the patient was found to be disoriented with a clouded sensorium. While the history of heavy daily alcohol use was clear in the chart and confirmed by other sources, it appeared unlikely that the patient had been using alcohol in the preceding month due to restricted access in his most recent living environment (a shared apartment with daily nursing assistance). He reported no lightheadedness, dizziness, palpitations, numbness, tingling, or any head trauma. He also negated the presence of active mood symptoms, auditory or visual hallucinations or suicidal ideation (SI).

The patient was admitted to the Internal Medicine Service and received a workup for the causes of delirium, including consideration of normal pressure hydrocephalus (NPH) and other neurologic conditions. Laboratory tests including a comprehensive metabolic panel, thyroid stimulating hormone, urinalysis, urine toxicology screen, and vitamin B12 and folate levels were in normal ranges. Although brain imaging revealed enlarged ventricles, NPH was considered unlikely because of the absence of ophthalmologic abnormalities, like gaze nystagmus, and urinary incontinence; conversely, there was some presence of urinary retention attributed to BPH and required an admission a few months prior. Moreover, magnetic resonance images showed that the ventricles were enlarged slightly out of proportion to the sulci, which can be seen with predominantly central volume loss compared with the pattern typically seen in NPH.

In light of concern for WE and the patient's history, treatment with IV thiamine and IV fluids was initiated and the Liaison Psychiatry Service was consulted for cognitive disability and treatment of his mood. Administration of IV thiamine rapidly restored his sensorium, but he became abruptly disorganized as the IV regimen graduated to an oral thiamine dose of $200 \mathrm{mg} 3$ times daily. Simultaneously, as medical stabilization was achieved, the patient was transferred to the inpatient psychiatry unit to address the nonresolving cognitive impairment and behavioral disorganization. This specifically involved newly emerging, impulsive, self-harming behaviors like throwing himself on the ground and banging his head on the floor. Such behaviors along with paucity of speech and decreased oral intake, ultimately warranted constant observation, which led to a decrease in self-harming activity. All this behavior was noted even though the patient was adherent to oral administration of thiamine. Throughout this time, the patient underwent several transfers back and forth between the Psychiatry and Internal Medicine services due to ongoing concern for the possibility of delirium or WE. However, the Neurology and Internal Medicine services did not feel that WE would explain the patient's mental and behavioral status, in part due to his ongoing adherence with daily oral thiamine dosing that was 
not associated with improvement in mental status.

Recollecting the patient's improvement with the parenteral thiamine regimen (IV and IM), the psychiatry unit tried a thiamine regimen of $200 \mathrm{mg}$ IM and $100 \mathrm{mg}$ oral 2 times daily. After about 2 weeks on this regimen, the patient subsequently achieved remarkable improvement in his cognitive and behavioral status, with resolution of selfharming behaviors. The patient was noted to be calmer, more linear, and more oriented, though he remained incompletely oriented throughout his hospitalization. As improvement in sensorium was established and the patient's hospital stay prolonged (Figure), his mood symptoms began manifesting as guilt, low energy, decreased appetite, withdrawal, and passive SI. This was followed by a trial of lithium that was discontinued due to elevated creatine levels. As the patient continued to report depression, a multidrug regimen of divalproex, fluoxetine, and quetiapine was administered, which lead to remarkable improvement.

At this time, it was concluded that the stores of thiamine in the patient's body may have been replenished, the alcohol intake completely ceased and that he needed to be weaned off of thiamine. The next step taken was reduction of the twice daily $200 \mathrm{mg} \mathrm{IM}$ thiamine dose to a once daily regimen, and oral thiamine was put on hold. Over the next 48 hours, the patient became less verbal, more withdrawn, incontinent of urine, and delirious. The twice daily IM $200 \mathrm{mg}$ thiamine was restarted, but this time the patient demonstrated very slow improvement. After 2 weeks, the IM thiamine $200 \mathrm{mg}$ was increased to 3 times daily, and the patient showed marked improvement in recall, mood, and effect.

Several attempts were made to reduce the IM thiamine burden on the patient and/ or transition to an exclusively oral regimen. However, he rapidly decompensated within hours of each attempt to taper the IM dose and required immediate reinstation. On the IM thiamine regimen, he eventually appeared to reach a stable cognitive and affective baseline marked by incomplete orientation but pleasant affect, he reported no mood complaints, behavioral stability, and an ability to comply with care needs and have simple con- versations. Some speech content remained disorganized particularly if engaged beyond simple exchanges.

The patient was discharged to a skilled nursing facility after a month of 3 times daily IM administration of thiamine. Within the next 24 hours, the patient returned to the ED with the originally reported symptoms of ataxia, agitation, and confusion. On inquiry, it was revealed that the ordered vials of IM thiamine for injection had not arrived with him at the nursing facility and he had missed 2 doses. The blood laboratory results, scans, and all other parameters were otherwise found to be normal and the patient was adherent to his prescribed antipsychotics and antidepressants. As anticipated, restoration of the IM thiamine regimen revived his baseline within hours. While confusion and delirium resolved completely with treatment, the memory impairments persisted. This patient has been administered a 3 times daily IM dose of $200 \mathrm{mg}$ thiamine for more than 2 years with a stable cognitive clinical picture.

\section{DISCUSSION}

According to data from the 2016 National Survey on Drug Use and Health, 16 million individuals in the US aged $\geq 12$ years reported heavy alcohol use, which is defined as binge drinking on $\geq 5$ days in the past month. ${ }^{6,7}$ Thiamine deficiency is an alcoholrelated disorder that is frequently encountered in hospital settings. This deficiency can also occur in the context of malabsorption, malnutrition, a prolonged course of vomiting, and bariatric surgery. 8,9

The deficiency in thiamine, which is sometimes known as WE, manifests rarely with all 3 of the classic triad of gait disturbances, abnormal eye movements, and mental status changes, with only $16.5 \%$ of patients displaying all of the triad. ${ }^{4}$ Moreover, there may be additional symptoms not listed in this triad, such as memory impairment, bilateral sixth nerve palsy, ptosis, hypotension, and hypothermia. ${ }^{10.11}$ This inconsistent presentation makes the diagnosis challenging and therefore requires a higher threshold for suspicion. If undiagnosed and/or untreated, WE can lead to chronic thiamine deficiency causing permanent brain damage in the guise of KS. This further increases the importance 
of timely diagnosis and treatment.

Our case highlights the utilization of an unconventional thiamine regimen that appeared to be temporally associated with mental status improvement. The patient's clouded sensorium and confusion could not be attributed to metabolic, encephalopathic, or infectious pathologies due to the absence of supportive laboratory evidence. He responded to IV and IM doses of thiamine, but repeated attempts to taper the IM doses with the objective of transitioning to oral thiamine supplementation were followed by immediate decompensations in mental status. This was atypical of WE as the patient seemed adequately replete with thiamine, and missing a few doses should not be enough to deplete his stores. Thus, reflecting a unique case of thiamine-dependent chronically set WE when even a single missed dose of thiamine adversely affected the patient's cognitive baseline. Interesting to note is this patient's memory issue, as evident by clinical examination and dating back at least 5 years as per chart review. This premature amnestic component of his presentation indicates a likely parallel running KS component of his presentation. Conversely, the patient's long history of alcohol use disorder, prior episodes of WE, and ideal response achieved only on parenteral thiamine repletion further supported the diagnosis of WE and our impression of the scenario.

Even though this patient had prior episodes of WE, there remained diagnostic uncertainty regarding his altered mental status for some time before the nonoral thiamine repletion treatment was implemented. Particularly in this admission, the patient's mental status frequently waxed and waned and there was the additional confusion of whether a potential psychiatric etiology contributed to some of the elements of his presentation, such as his impulsive self-harm behaviors. This behavior led to recurrent transfers among the Psychiatry Service, Internal Medicine Service, and the ED.

The patient's presentation did not reflect the classical triad of WE, and while this is consistent with the majority of clinical manifestations, various services were reluctant to attribute his symptoms to WE. Once the threshold of suspicion of thiamine deficiency was lowered and the deficit treated more ag- gressively, the patient seemed to improve tremendously. Presence of memory problems and confabulation, both of which this patient exhibited, are suggestive of KS and are not expected to recover with treatment, yet for this patient there did seem to be some improvement-though not complete resolution. This is consistent with newer evidence suggesting that some recovery from the deficits seen in $\mathrm{KS}$ is possible. ${ }^{3}$

Once diagnosed, the treatment objective is the replenishment of thiamine stores and optimization of the metabolic scenario of the body to prevent recurrence. For acute WE symptoms, many regimens call for 250 to $500 \mathrm{mg}$ of IV thiamine supplementation 2 to 3 times daily for 3 to 5 days. High dose IV thiamine ( $\geq 500 \mathrm{mg}$ daily) has been proposed to be efficacious and free of considerable adverse effects. ${ }^{12}$ A study conducted at the University of North Carolina described thiamine prescribing practices in a large academic hospital, analyzing data with the objective of assessing outcomes of ordering high-dose IV thiamine (HDIV, $\geq 200 \mathrm{mg}$ IV twice daily) to patients with encephalopathy..$^{13}$ The researchers concluded that HDIV, even though rarely prescribed, was associated with decreased inpatient mortality in bivariable models. However, in multivariable analyses this decrease was found to be clinically insignificant. Our patient benefitted from both IV and IM delivery.

Ideally, after the initial IV thiamine dose, oral administration of thiamine 250 to $1,000 \mathrm{mg}$ is continued until a reduction, if not abstinence, from alcohol use is achieved..$^{5}$ Many patients are discharged on an oral maintenance dose of thiamine $100 \mathrm{mg}$. Oral thiamine is poorly absorbed and less effective in both prophylaxis and treatment of newly diagnosed WE; therefore, it is typically used only after IM or IV replenishment. It remains unclear why this patient required IM thiamine multiple times per day to maintain his mental status, and why he would present with selfinjurious behaviors after missing doses. The patient's response can be attributed to late-onset defects in oral thiamine absorption at the carrier protein level of the brush border and basolateral membranes of his jejunum; however, an invasive procedure like a jejunal biopsy to establish the 
definitive etiology was neither necessary nor practical once treatment response was observed. ${ }^{14}$ Other possible explanations include rapid thiamine metabolism, poor gastrointestinal absorption and a late-onset deficit in the thiamine diffusion mechanisms, and active transport systems (thiamine utilization depends on active transport in low availability states and passive transport when readily available). The nature of these mechanisms deserves further study. Less data have been reported on the administration and utility of IM thiamine for chronic WE; hence, our case report is one of the first illustrating the role of this method for sustained repletion.

\section{CONCLUSIONS}

This case presented a clinical dilemma because the conventional treatment regimen for WE didn't yield the desired outcome until the mode and duration of thiamine administration were adjusted. It illustrates the utility of a sustained intensive thiamine regimen irrespective of sobriety status, as opposed to the traditional regimen of parenteral (primarily IV) thiamine for 3 to 7 days, followed by oral repletion until the patient achieves sustained abstinence. In this patient's case, access to nursing care postdischarge facilitated his continued adherence to IM thiamine therapy.

The longitudinal time course of this case suggests a relationship between this route of administration and improvement in symptom burden and indicates that this patient may have a long-term need for IM thiamine to maintain his baseline mental status. Of great benefit in such patients would be the availability of a long-acting IM thiamine therapy. Risk of overdose is unlikely due to the water solubility of B group vitamins.

This case report highlights the importance of setting a high clinical suspicion for WE due to its ever-increasing incidence in these times. We also wish to direct researchers to consider other out-of-the-box treatment options in case of failure of the conventional regime. In documenting this patient report, we invite more medical providers to investigate and explore other therapeutic options for WE treatment with the aim of decreasing both morbidity and mortality secondary to the condition.

\section{Author disclosures}

The authors report no actual or potential conflicts of interest with regard to this article.

\section{Disclaimer}

The opinions expressed herein are those of the authors and do not necessarily reflect those of Federal Practitioner, Frontline Medical Communications Inc., the US Government, or any of its agencies. This article may discuss unlabeled or investigational use of certain drugs. Please review the complete prescribing information for specific drugs or drug combinations -including indications, contraindications, warnings, and adverse effects-before administering pharmacologic therapy to patients.

\section{References}

1. Lough ME. Wernicke's encephalopathy: expanding the diagnostic toolbox. Neuropsychol Rev. 2012;22(2):181-194. doi:10.1007/s11065-012-9200-7

2. Arts NJ, Walvoort SJ, Kessels RP. Korsakoff's syndrome: a critical review. Neuropsychiatr Dis Treat. 2017;13:28752890. Published 2017 Nov 27. doi:10.2147/NDT.S130078

3. Johnson JM, Fox V. Beyond thiamine: treatment for cognitive impairment in Korsakoff's syndrome. Psychosomatics. 2018;59(4):311-317. doi:10.1016/j.psym.2018.03.011

4. Harper CG, Giles M, Finlay-Jones R. Clinical signs in the Wernicke-Korsakoff complex: a retrospective analysis of 131 cases diagnosed at necropsy. J Neurol Neurosurg Psychiatry. 1986;49(4):341-345. doi:10.1136/ jnnp.49.4.341

5. Xiong GL, Kenedl, CA. Wernicke-Korsakoff syndrome. https://emedicine.medscape.com/article/288379-overview. Updated May 16, 2018, Accessed July 24, 2020.

6. Ahrnsbrak R, Bose J, Hedden SL, Lipari RN, Park-Lee E. Results from the 2016 National Survey on Drug Use and Health. https://www.samhsa.gov/data/sites/default/files /NSDUH-FFR1-2016/NSDUH-FFR1-2016.htm. Accessed July 22, 2020.

7. National Institute on Alcohol Abuse and Alcoholism. Drinking Levels Defined. https://www.niaaa.nih.gov /alcohol-health/overview-alcohol-consumption/moderate -binge-drinking Accessed July 24, 2020.

8. Heye N, Terstegge K, Sirtl C, McMonagle U, Schreiber K, Meyer-Gessner M. Wernicke's encephalopathy--causes to consider. Intensive Care Med. 1994;20(4):282-286. doi:10.1007/BF01708966

9. Aasheim ET. Wernicke encephalopathy after bariatric surgery: a systematic review. Ann Surg. 2008;248(5):714-720. doi:10.1097/SLA.0b013e3181884308

10. Victor M, Adams RD, Collins GH. The Wernicke-Korsakoff Syndrome and Related Neurologic Disorders Due to Alcoholism and Malnutrition. Philadelphia, PA: FA Davis; 1989.

11. Thomson AD, Cook CC, Touquet R, Henry JA; Royal College of Physicians, London. The Royal College of Physicians report on alcohol: guidelines for managing Wernicke's encephalopathy in the accident and Emergency Department [published correction appears in Alcohol Alcohol. 2003 May-Jun;38(3):291]. Alcohol Alcohol. 2002;37(6):513-521. doi:10.1093/alcalc/37.6.513

12. Nishimoto A, Usery J, Winton JC, Twilla J. High-dose parenteral thiamine in treatment of Wernicke's encephalopathy: case series and review of the literature. In Vivo. 2017;31(1):121-124. doi:10.21873/invivo.11034

13. Nakamura ZM, Tatreau JR, Rosenstein DL, Park EM. Clinical characteristics and outcomes associated with highdose intravenous thiamine administration in patients with encephalopathy. Psychosomatics. 2018;59(4):379-387. doi:10.1016/j.psym.2018.01.004

14. Subramanya SB, Subramanian VS, Said HM. Chronic alcohol consumption and intestinal thiamin absorption: effects on physiological and molecular parameters of the uptake process. Am J Physiol Gastrointest Liver Physiol. 2010;299(1):G23-G31. doi:10.1152/ajpgi.00132.2010 